\title{
Adaptive model predictive control for co-ordination of compression and friction brakes in heavy duty vehicles
}

\author{
Ardalan Vahidi ${ }^{1, *, \dagger}$, Anna Stefanopoulou ${ }^{2, \ddagger}$ and Huei Peng ${ }^{2, \S}$ \\ ${ }^{1}$ Department of Mechanical Engineering, Clemson University, 208 Fluor Daniel Building, \\ Clemson, SC 29634, U.S.A. \\ ${ }^{2}$ Department of Mechanical Engineering, University of Michigan, Ann Arbor, MI, U.S.A.
}

\begin{abstract}
SUMMARY
In this paper, an adaptive model predictive control scheme is designed for speed control of heavy vehicles. The controller co-ordinates use of compression brakes and friction brakes on downhill slopes. Moreover, the model predictive controller takes the actuator constraints into account. A recursive least square scheme with forgetting is used in parallel with the controller to update the estimates of vehicle mass and road grade. The adaptation improved the model predictive controller. Also online estimation of the road grade enhanced the closed-loop performance further by contributing through feedforward control. Simulations of realistic driving scenarios with a validated longitudinal vehicle model are used throughout this paper to illustrate the benefits of co-ordinating the two braking mechanisms and influence of unknown vehicle mass and road grade. Copyright (C) 2006 John Wiley \& Sons, Ltd.
\end{abstract}

Received 13 January 2006; Revised 10 May 2006; Accepted 31 May 2006

KEY WORDS: adaptive control; mass and grade estimation; compression braking; vehicle control; model predictive control

\section{INTRODUCTION}

During the past decade much research has been dedicated to automation of longitudinal control of vehicles $[1,2]$. Particular attention is given to heavy duty vehicles. Specific travelling routes and centralized fleet management allows automation of heavy vehicle with significant impact on

\footnotetext{
*Correspondence to: Ardalan Vahidi, Department of Mechanical Engineering, Clemson University, 208 Fluor Daniel Building, Clemson, SC 29634, U.S.A.

†E-mail: avahidi@clemson.edu

ĐE-mail: annastef@umich.edu

${ }^{\S}$ E-mail: hpeng@umich.edu

Contract/grant sponsor: California Department of Transportation; contract/grant number: TO 4234
} 
transportation networks. The large mass of a heavy duty vehicle demands large braking forces during deceleration and results in frequent actuator saturations during braking. Large delays associated with the pneumatic actuation in heavy vehicles friction brakes impose additional limits in their use [3]. Therefore, other retarding mechanisms like compression braking or transmission retarders are common in heavy trucks. In the compression braking mode, the engine is used as a compressor that absorbs kinetic energy from the crankshaft. Compression braking is a standard mechanism on today's heavy vehicles and is a suitable actuator in speed control applications. For example, in Eaton-Vorad collision-warning system with SmartCruise EVT-V300, compression braking is automatically activated when a collision is imminent [4].

In the existing compression braking systems, the retarding power level is quantized and depends on the engine speed and a selected number of cylinders activated by the driver. For example two, four or six cylinders can be activated to generate three levels of retarding power for a certain engine speed [4]. While successful implementation of the discrete compression brake mechanism in automated longitudinal control has been demonstrated in experiments [4], more flexibility is expected with the more advanced continuously variable compression braking systems. A continuous spectrum of retarding powers can be achieved in a camless engine and by variable valve timing. A detailed crank angle based model of such a continuous compression brake system is developed in Reference [5] and validated with experimental data from engine dynamometer. In Reference [6] a non-linear Lyapunov-based control design has been proposed for continuously variable compression braking system in longitudinal control; and a reference velocity governor which prevents the saturation of braking actuators. To compensate for large parameter variations typical to heavy vehicles, a model reference adaptive algorithm is proposed in Reference [7] which uses a continuous compression braking system. So Reference [6] addresses possible actuator saturations and Reference [7] treats vehicle parameter variation. In this paper, we address both difficulties simultaneously.

Constrained optimization is necessary to make the best use of compression brakes and friction brakes given their saturation limits. This goal can be achieved by a model predictive control design. In this paper, we design an adaptive model predictive controller for co-ordination of the compression brakes and friction brakes. MPC takes the actuator constraints into account, when calculating the control command. Moreover addition of a parameter estimation scheme, improves the performance of the controller when there are uncertainties in vehicle mass. Estimation of the road grade disturbance enhances the performance further, by contributing to feedforward control. The proposed methodology can be used in automated longitudinal control applications such as platooning and adaptive cruise control. Also in manual driving, the brake-by-wire system can use both retarding mechanisms to achieve the requested deceleration. In the next section, we summarize the longitudinal dynamics model of the vehicle and the braking systems. Then an adaptive model predictive control methodology is briefly explained. Simulation results are accompanied by analysis on performance of the controller in presence of actuator constraints and parameter uncertainties.

\section{LONGITUDINAL DYNAMICS MODEL}

Assuming only moderate braking and no wheel slip, which is a good assumption at highway cruise speeds, the longitudinal dynamics equation of the vehicle can be written 
as follows:

$$
M \dot{v}=\left(-T_{\mathrm{cb}}-J_{e} \dot{\omega}\right) / r_{g}-T_{\mathrm{fb}} / r_{\mathrm{w}}-F_{\mathrm{a}}-F_{g}
$$

In this equation, $M$ is the total mass of the vehicle, $J_{e}$ is the driveline inertia, $v$ is the velocity and $\omega$ is rotational engine speed. Total friction braking torque at the wheels is represented by $T_{\mathrm{fb}}$ and $T_{\mathrm{cb}}$ is the compression braking torque at the flywheel. The assumption is that transmission and the torque converter are fully engaged and the torque is passed to the wheels without any loss. Parameter $r_{g}$ is defined as $r_{g}=r_{\mathrm{w}} /\left(g_{\mathrm{d}} g_{\mathrm{f}}\right)$, where $r_{\mathrm{w}}$ is the wheel radius, $g_{\mathrm{d}}$ is the gear ratio and $g_{\mathrm{f}}$ is the final drive ratio. The aerodynamic resistance is given by $F_{\mathrm{a}}=0.5 C_{\mathrm{d}} \rho A v^{2}$ where $C_{\mathrm{d}}$ is the drag coefficient, $\rho$ is air density and $A$ is frontal area of the vehicle. The combined force due to road grade $(\beta)$ and the rolling resistance between tire and road $\left(c_{\mathrm{rr}}\right)$ is given by $F_{g}=M g\left(c_{\mathrm{rr}} \cos \beta+\sin \beta\right)$, where $g$ is the gravity constant. Here $\beta=0$ corresponds to no inclination, $\beta>0$ corresponds to uphill grade and $\beta<0$ represents downhill.

For linear control design, Equation (1) can be linearized around a nominal operating velocity $v^{o}$ :

$$
M \delta \dot{v}=\left(-\delta T_{\mathrm{cb}}-J_{e} \delta \dot{\omega}\right) / r_{g}-\delta T_{\mathrm{sb}} / r_{\mathrm{w}}-C_{\mathrm{d}} \rho A v^{o} \delta v-\delta F_{g}
$$

The operator, $\delta$, represents deviation of each variable from the equilibrium values. Choosing a sampling time of $T_{\mathrm{s}}$, the above equation can be discretized as follows:

$$
\delta v(k+1)=c_{1} \delta v(k)+c_{2}\left(-\delta T_{\mathrm{cb}}(k) / r_{g}-\delta T_{\mathrm{sb}}(k) / r_{\mathrm{w}}-\delta F_{g}(k)\right)
$$

where

$$
\begin{aligned}
& c_{1}=1-\left(2 T_{\mathrm{s}} k_{\mathrm{a}} v^{o}\right) / M_{\mathrm{eff}}, \quad c_{2}=T_{\mathrm{s}} / M_{\mathrm{eff}} \\
& k_{\mathrm{a}}=0.5 C_{\mathrm{d}} \rho A, \quad M_{\mathrm{eff}}=M+J_{e} / r_{g}^{2}
\end{aligned}
$$

For the control purpose, velocity of the vehicle, $v$, engine speed, $\omega$, compression braking torque, $T_{\mathrm{cb}}$ and gear number, $g_{\mathrm{d}}$, can be obtained from vehicle's control area network (CAN). The friction brake torque is normally hard to measure but can be estimated based on the brake model presented later in this chapter. The mass is either known or is estimated using an estimation scheme. Other vehicle parameters like wheel radius and gear ratios are fixed values which are normally known in advance. In general, road grade is unknown and therefore acts as an unmeasured disturbance to the system. However it can be determined using an online estimation scheme or using GPS co-ordinates combined with a digital map of the road. Next we will explain the dynamic models of compression brake and friction brakes used in this study.

\subsection{Compression brake model}

For control design purpose, the dynamics of the compression braking event can be modelled by a first-order lag:

$$
\dot{T}_{\mathrm{cb}}(t)=-\frac{1}{\tau_{\mathrm{cb}}}\left(T_{\mathrm{cb}}(t)-T_{\mathrm{cb}}^{\mathrm{st}}(t)\right)
$$

where $\tau_{\mathrm{cb}}$ is the compression brake time constant. The engine braking torque at steady state, $T_{\mathrm{cb}}^{\mathrm{st}}$, can be specified as a non-linear function of engine speed and brake valve opening as follows:

$$
T_{\mathrm{cb}}^{\mathrm{st}}(\omega, \mathrm{BVO})=\alpha_{0}+\alpha_{1} \omega+\alpha_{2} \mathrm{BVO}+\alpha_{3} \omega \mathrm{BVO}
$$


where BVO is the brake valve opening in crank angle degrees. "The coefficients, $\alpha_{i}$, are derived in Reference [8] based on a detailed model of a continuous compression brake. When $T_{\mathrm{cb}}$ is in Nm, $\mathrm{BVO}$ is in degrees and $\omega$ is in $\mathrm{rad} / \mathrm{s}$, the parameters of the model are:

$$
\alpha_{0}=-1893, \quad \alpha_{1}=48.13, \quad \alpha_{2}=2.8588, \quad \alpha_{3}=-0.07839
$$

Equation (4) is linearized around the operating point and then discretized with a sampling time of $T_{\mathrm{s}}$ to yield:

$$
\delta T_{\mathrm{cb}}(k+1)=\left(1-\frac{T_{\mathrm{s}}}{\tau_{\mathrm{cb}}}\right) \delta T_{\mathrm{cb}}(k)+\frac{T_{\mathrm{s}}}{\tau_{\mathrm{cb}}} \delta T_{\mathrm{cb}}^{\mathrm{st}}
$$

where the operator, $\delta$, shows deviation from the operating point. The nominal downhill grade is chosen to be $\beta^{o}=-1.25^{\circ}$. At equilibrium, we assume that the truck is in fourth gear, cruising at $v^{o}=20 \mathrm{~m} / \mathrm{s}$. Using the parameters of a $\mathrm{PATH}^{\|}$experimental truck, the total driveline ratio, $r_{g}^{o}$, for the fourth gear is 0.1102 . Therefore, the operating engine speed is

$$
\omega^{o}=v^{o} / r_{g}^{o}=181.47 \mathrm{rad} / \mathrm{s} \approx 1800 \mathrm{rpm}
$$

We assume that this constant speed is maintained by application of compression brakes only and friction brakes are not applied at this equilibrium point. Using (5), the nominal brake valve timing which corresponds to this operating point is $\mathrm{BVO}^{\circ}=650^{\circ}$. We define the compression brake control command, $u_{\mathrm{cb}}$, to be the deviation from this nominal value: $u_{\mathrm{cb}}=\mathrm{BVO}-\mathrm{BVO}^{o}$. The linearized model for steady-state engine brake torque is then:

$$
\delta T_{\mathrm{cb}}^{\mathrm{st}}=2.82 \delta \omega+11.36 u_{\mathrm{cb}}
$$

A schematic of a compression brake event is shown in Figure 1. As shown in the figure the operating range for brake valve opening is between 620 and $680^{\circ}$. Therefore, upper and lower hard constraints on $u_{\mathrm{cb}}$ are

$$
-30 \leqslant u_{\mathrm{cb}} \leqslant 30
$$

\subsection{Service brake model}

A model of the service brakes can be obtained by using a linear static model and a first-order lag which represents the dynamics. Since we use the friction brakes moderately, only to compensate for torque deficits, uncertainties due to brake overheating and saturations will not be considered here. We assume that the braking torque is available at four wheels. The dynamics of the brake is modelled by a first-order lag:

$$
\dot{T}_{\mathrm{sb}}(t)=-\frac{1}{\tau_{\mathrm{sb}}}\left(T_{\mathrm{sb}}(t)-T_{\mathrm{sb}}^{\mathrm{st}}(t)\right)
$$

\footnotetext{
"In compression braking, the vehicle kinetic energy is absorbed by compressing the cylinder air charge. The secondary opening of the exhaust valve at the end of compression stroke releases the compressed air charge to the exhaust manifold. This secondary opening of the exhaust valve is called the brake valve opening (BVO) event and is characterized by the crank angle degrees from top-dead-centre (TDC) as shown in Figure 1 see Reference [8] for details. " Partners for Advanced Transit and Highways.
} 


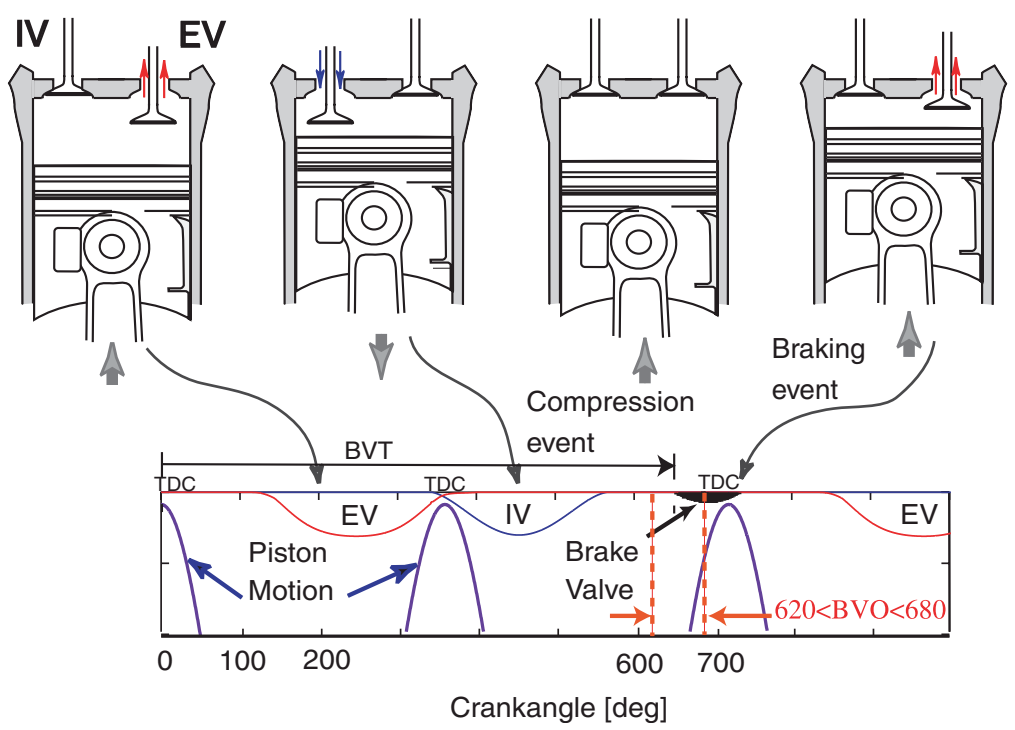

Figure 1. Schematic of the compression brake event. Modified graphics from Reference [7].

where $\tau_{\mathrm{sb}}$ is the friction brake time constant. The static braking torque, $T_{\mathrm{sb}}^{\mathrm{st}}$, at the wheel is proportional to the brake command, $u_{\mathrm{sb}}$ :

$$
T_{\mathrm{sb}}^{\mathrm{st}}=\mu_{\mathrm{d}} r_{\mathrm{d}} A_{\mathrm{d}} \kappa_{\mathrm{s}} u_{\mathrm{sb}}
$$

where $\mu_{\mathrm{d}}$ is the friction coefficient between the brake drum and brake pad, $r_{\mathrm{d}}$ is the brake drum radius and $A_{\mathrm{d}}$ is area of diaphragm, $\kappa_{\mathrm{s}}$ is determined by the manufacturer and relates the command input, $u_{\mathrm{sb}}$, to the pressure on the brake diagram [9]. The values were experimentally identified for an experimental Freightliner truck by PATH researchers. The lumped coefficient from the command voltage to total braking torque was determined to be $272.5 \mathrm{Nm} / \mathrm{V}$ [10]. Given that the nominal friction brake torque, $T_{\mathrm{sb}}^{o}$, is zero, we can write:

$$
\delta T_{\mathrm{sb}}^{\mathrm{st}}=272.5 u_{\mathrm{sb}}
$$

The range for command voltage of the experimental truck was $0-10 \mathrm{~V}$. However, it was determined that at five volts the wheels would lockup. Therefore, we constrain the command voltage between 0 and $5 \mathrm{~V}$ :

$$
0 \leqslant u_{\mathrm{sb}} \leqslant 5
$$

The discrete form of (9) is

$$
\delta T_{\mathrm{sb}}(k+1)=\left(1-\frac{T_{\mathrm{s}}}{\tau_{\mathrm{sb}}}\right) \delta T_{\mathrm{sb}}(k)+\frac{T_{\mathrm{s}}}{\tau_{\mathrm{sb}}} \delta T_{\mathrm{sb}}^{\mathrm{st}}
$$

where the operator, $\delta$, shows deviation from the operating point. Co-ordination of the friction brakes with compression brakes is based on a model predictive control design which is explained next. 


\section{MODEL PREDICTIVE CONTROL DESIGN}

A brief summary of the specific MPC design, used in this work is provided in this section. For more details see Reference [11].

We define the state vector as $x=\left[\begin{array}{lll}\delta v(k) & \delta T_{\mathrm{cb}}(k) & \delta T_{\mathrm{sb}}(k)\end{array}\right]^{\mathrm{T}}$ and combine Equations (3), (6) and (13) to get:

$$
\begin{aligned}
x(k+1) & =A x(k)+B_{u} u(k)+B_{w} w(k) \\
y(k) & =C x(k)
\end{aligned}
$$

where the control inputs are compression brake and friction brake commands:

$$
u(k)=\left[\begin{array}{ll}
u_{\mathrm{cb}}(k) & u_{\mathrm{sb}}(k)
\end{array}\right]^{\mathrm{T}}
$$

and the disturbance is

$$
w(k)=-M g\left(\left(c_{\mathrm{rr}} \cos \beta+\sin \beta\right)-\left(c_{\mathrm{rr}} \cos \beta^{0}+\sin \beta^{0}\right)\right)
$$

and $\beta$ is the total road grade, $\beta^{0}$ is the nominal grade. The system matrices are:

$$
\begin{aligned}
A & =\left[\begin{array}{ccc}
1-\frac{2 T_{\mathrm{s}} k_{\mathrm{a}} v^{o}}{M_{\mathrm{eff}}} & -\frac{T_{\mathrm{s}}}{r_{g} M_{\mathrm{eff}}} & -\frac{T_{\mathrm{s}}}{r_{\mathrm{w}} M_{\mathrm{eff}}} \\
\frac{2.82 T_{\mathrm{s}}}{r_{g} \tau_{\mathrm{cb}}} & 1-\frac{T_{\mathrm{s}}}{\tau_{\mathrm{cb}}} & 0 \\
0 & 0 & 1-\frac{T_{\mathrm{s}}}{\tau_{\mathrm{sb}}}
\end{array}\right] \\
B_{u} & =\left[\begin{array}{cc}
0 & 0 \\
11.36 \frac{T_{\mathrm{s}}}{\tau_{\mathrm{cb}}} & 0 \\
0 & 272.5 \frac{T_{\mathrm{s}}}{\tau_{\mathrm{sb}}}
\end{array}\right] \quad B_{w}=\left[\begin{array}{c}
\frac{T_{\mathrm{s}}}{M_{\mathrm{eff}}} \\
0 \\
0
\end{array}\right]
\end{aligned}
$$

We wish to regulate the velocity and minimize the use of friction brake when possible to reduce the brake wear. To achieve this goal, velocity and friction brake torques are chosen as performance variables:

$$
y(k)=\left[\begin{array}{lll}
\delta v(k) & \delta T_{\mathrm{sb}}(k)
\end{array}\right]
$$

Therefore the measurement vector is:

$$
C=\left[\begin{array}{lll}
1 & 0 & 0 \\
0 & 0 & 1
\end{array}\right]
$$

The velocity is available from the control area network (CAN) [12]. Friction brake torque can be estimated based on brake pressure measured at wheels. For instance, the PATH experimental truck was equipped with pressure transducers at each wheel that give an estimate of pneumatic 
brake's air pressure [9]. The sensed pressure $\hat{P}_{\mathrm{sb}}$ can be translated into an estimate of friction brake torque $\hat{T}_{\mathrm{sb}}$ using the static relationship $\hat{T}_{\mathrm{sb}}=\mu_{\mathrm{d}} r_{\mathrm{d}} A_{\mathrm{d}} \hat{P}_{\mathrm{sb}}$. Therefore, all the performance variables are treated as measured variables in this analysis.

The future outputs of the plant can be predicted based on (14):

$$
\begin{aligned}
& \hat{x}(k+1 \mid k)=A \hat{x}(k \mid k-1)+B_{u} u(k)+B_{w} w(k) \\
& \hat{y}(k \mid k-1)=C \hat{x}(k \mid k-1)
\end{aligned}
$$

where $\hat{x}(k+1 \mid k)$ is the estimate of the state at future sampling instant, $k+1$, based on information available at instant $k$, and $\hat{y}(k \mid k-1)$ is the output estimate at instant $k$ based on information available at instant $k-1$. If the road grade is unknown the disturbance, $w$, is assumed to be zero in the future prediction horizon. If the road grade is estimated at each instant $k$, then $w(k)$ is known and we assume it stays constant in the next prediction horizon. In this paper we consider both scenarios. ${ }^{* *}$ By recursive use of Equation (16), the future outputs $\hat{y}(k+j \mid k)$ can be predicted based on information available at the present instant.

In MPC, we seek a control sequence of size $N$ :

$$
u^{N}=\left[\begin{array}{llll}
u(k) & u(k+1) & \cdots & u(k+N-1)
\end{array}\right]^{\mathrm{T}}
$$

that minimizes the deviation of predicted outputs from their reference values, over the future horizon of $P$ sampling times $(N<P)$. The following finite horizon cost function is defined:

$$
J(k)=\sum_{j=1}^{P}\left(\|(r(k)-\hat{y}(k+j \mid k))\|_{Q}^{2}+\|\Delta u(k+j-1)\|_{S}^{2}\right)
$$

where $S$ and $Q$ are input and output weighting matrices, respectively, and $r$ is the reference to be tracked. The reference friction brake torque is zero. The reference velocity is determined by a higher level supervisory control in automated longitudinal control [13] or by the driver in cruise control mode. In the model predictive control design the assumption is that the reference stays constant over each prediction horizon. The control sequence, $u^{N}$, should minimize the performance index shown above and also satisfy the compression and friction brake constraints described by (8) and (12):

$$
\left[\begin{array}{ll}
-30 & 0
\end{array}\right]^{\mathrm{T}} \leqslant u(k+j) \leqslant\left[\begin{array}{ll}
30 & 5
\end{array}\right]^{\mathrm{T}}, \quad j=0,1, \ldots, N-1
$$

Furthermore we impose slew rate constraints on the inputs, to avoid unrealistic variations of the input command. In simulations of this paper, we used the following rate constraints:

$$
\left[\begin{array}{ll}
-5 & -0.5
\end{array}\right]^{\mathrm{T}} \leqslant \Delta u(k+j) \leqslant\left[\begin{array}{ll}
5 & 0.5
\end{array}\right]^{\mathrm{T}}, \quad j=0,1, \ldots, N-1
$$

The predicted output, $\hat{y}(k+j \mid k)$, can be calculated as a function of the control sequence, $u^{N}$. The performance index (17) and constraints (18) and (19) can be written as functions of $u^{N}$ and measured outputs, disturbances and the reference command in a quadratic form. Quadratic programming techniques could be used to solve this constrained optimization problem at each sampling time. In absence of constraints, the problem reduces to a simple minimization problem

\footnotetext{
**A Another possible scenario is when preview information of the upcoming road grade is available through GPS. We do not consider this latter case in this paper.
} 
and an explicit control law can be calculated. With constraints, on the other hand, a straightforward explicit control law does not exist. Instead numerical optimization of the performance index is carried out online to find the control input. Simulating a constrained problem normally takes much longer time than the equivalent unconstrained problem. Since the solution depends on iterative numerical procedure, as the constraints become more stringent the computational time increases. So pushing the system to its limits for the best possible performance, might result in large computational time. Therefore, a balance between tightening the constraints and computational resources is necessary.

Selection of prediction horizon, penalty weights, and influence of mass and grade uncertainty are explained with simulation analysis, next. An adaptive scheme, which enhances the closedloop performance is discussed in the final part of the paper.

\section{SIMULATION RESULTS: NO ADAPTATION}

In this paper, the closed-loop performance is studied for step deviations of the grade from the nominal point of $-1.25^{\circ}$. The desired velocity is fixed at $20 \mathrm{~m} / \mathrm{s}$. The sampling frequency is $10 \mathrm{~Hz}$ in all simulations. In practice the control horizon can be chosen smaller than the prediction horizon to reduce the number of optimization variables which saves computational time. Here we have a low-order model and we do not expect the computational time to be an issue, therefore we select the length of the control horizon $N$, equal to the prediction horizon, $P$. This will reduce the number of design parameters. The length of the prediction horizon influences the stability margin and was determined in early stage of design by simulation analysis using the linearized vehicle model. Figure 2 shows the loci of the closed-loop poles in the $z$-plane, as the prediction horizon $P H$ is increased from 2 sampling steps to 50 sampling steps. As seen in the graph, the system is more damped for longer horizons. Figure 2 shows that a prediction horizon above 10 steps only slightly helps the damping ratio of the poles but increases the computation time. Therefore, we fix the prediction horizon at 10 sampling steps for the rest of simulations.

Consider $Q=\operatorname{diag}\left(Q_{v}, Q_{T \mathrm{sb}}\right)$ and $S=\operatorname{diag}\left(S_{\mathrm{cb}}, S_{\mathrm{sb}}\right)$ in the performance index (17), where $Q_{v}, Q_{T \mathrm{sb}}, S_{\mathrm{cb}}$ and $S_{\mathrm{sb}}$ are penalties on velocity error, friction brake torque, compression brake input and friction brake command input, respectively. Since only the relative value of the weights with respect to each other determines the penalty on each performance variable we fixed the penalty on velocity error at 1 . Since we expect the friction brake torque to be in the order of $0-500 \mathrm{~N} \mathrm{~m}$ and the velocity deviation to be in the order of $0-2 \mathrm{~m} / \mathrm{s}$ we chose the penalty on friction brake torque to be roughly $(2 / 500)^{2} \approx 2 \times 10^{-5}$; this choice will normalize the performance variables to be of the same order. We then determined the two remaining weights graphically: Figure 3 shows the influence of these weights on root mean square (RMS) velocity error and RMS friction brake torque for a step change in road grade. In each plot, the $x$-axis shows the penalty on friction brake command and each curve corresponds to a different penalty on compression brake input. The input constraints are also enforced. Based on this plot, we found that weights of 0.01 on the compression brake input and 0.1 on friction brake input result in acceptable velocity regulation. This choice of weights penalizes the use of friction brakes more than compression brakes and therefore reduces the friction brake use when feasible. As a result the friction brake wear is minimized. Figure 4 shows the performance of the controller during the sequence of step changes in road grade. As 


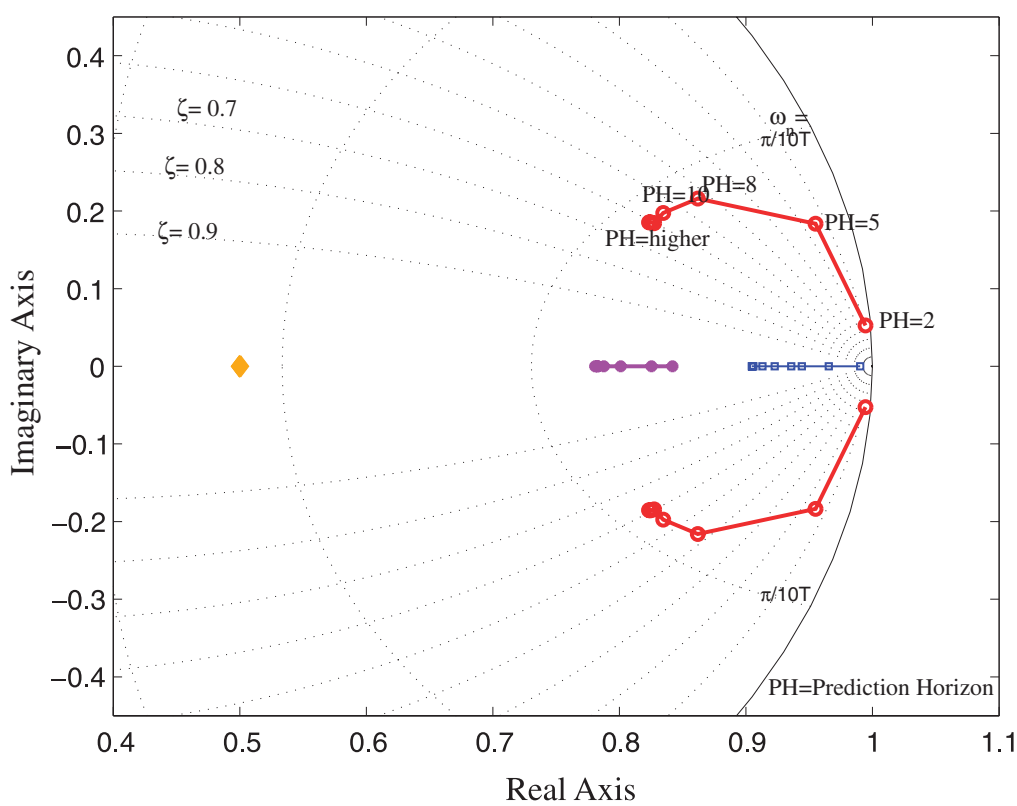

Figure 2. Loci of closed-loop poles in $z$-domain as prediction horizon increases from $\mathrm{PH}=2$ to $\mathrm{PH}=50$ steps. The performance index weights are $Q=\operatorname{diag}(1,0.00002), S=\operatorname{diag}(0.01,0.1)$.
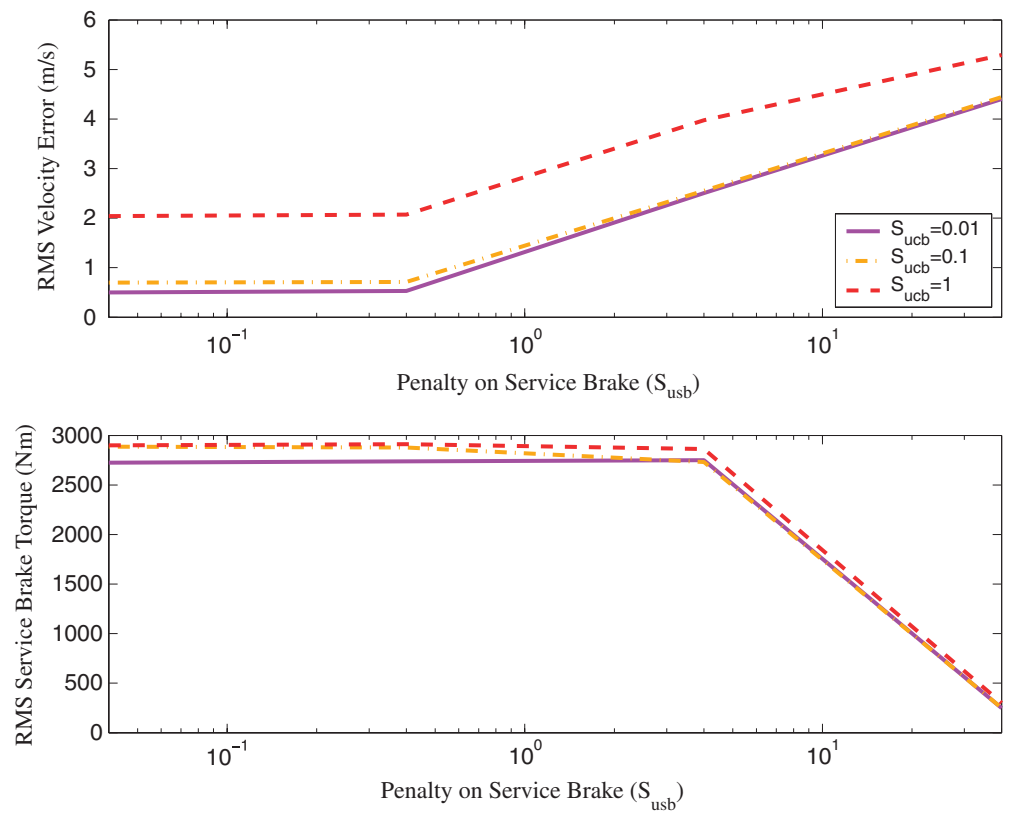

Figure 3. RMS error in velocity and RMS friction brake torque for different penalties on each brake use. Input constraints are enforced. 

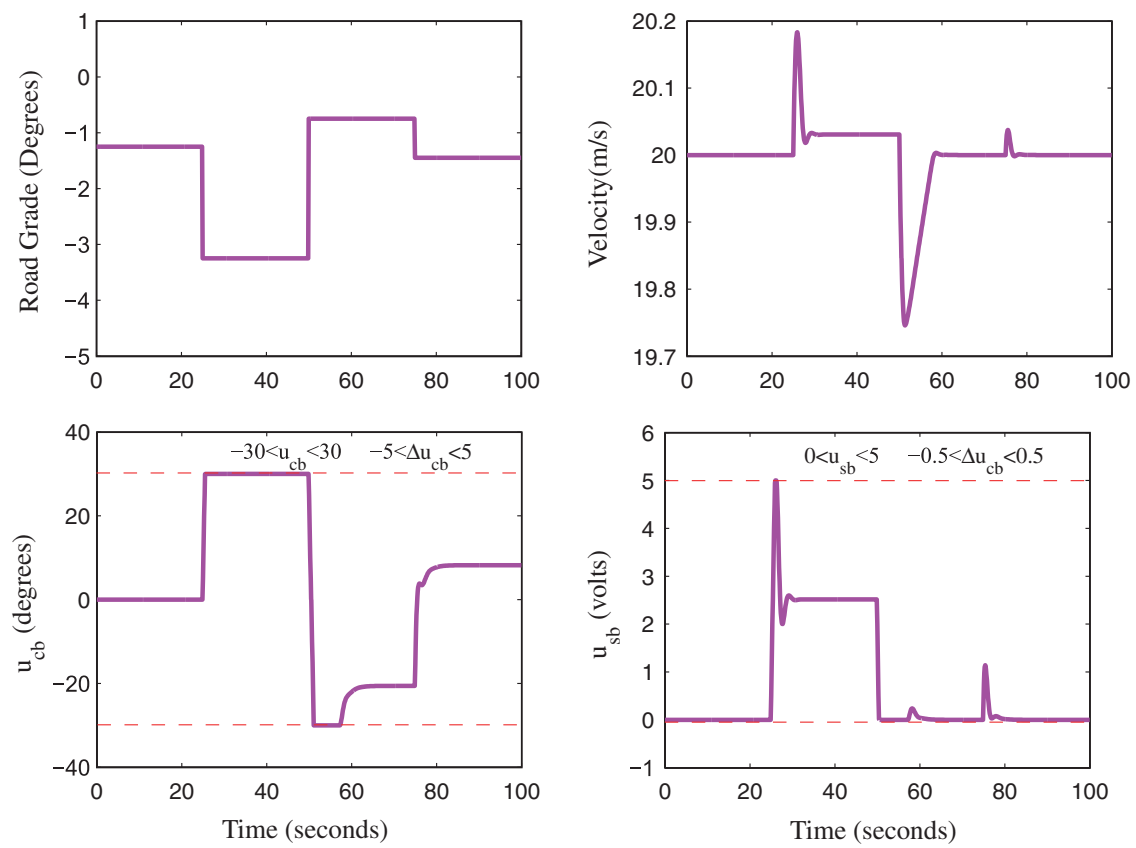

Figure 4. Velocity regulation with input and input rate constraints. The total vehicle mass is $25000 \mathrm{~kg}$. The performance index weights are $Q=\operatorname{diag}(1,0.00002), S=\operatorname{diag}(0.01,0.1)$.

shown in this figure, the friction brakes are used only after the compression brake is fully utilized.

In the above analysis, we assumed that vehicle mass was known and therefore there was no discrepancy between the plant and model. Furthermore, we assumed that the road grade is measured and is used in feedforward control at each sampling time. However, mass and grade are unknown in most realistic driving scenarios. Road grade measurement requires additional sensors which add to the cost of the vehicle. Also in heavy duty vehicles, the mass can vary largely from a configuration of no payload to full payload. Therefore, a longitudinal controller that is tuned for a particular mass, might be too aggressive or too slow for a different mass. A controller that is designed for the unloaded $9000 \mathrm{~kg}$ truck, will be sluggish in controlling the truck with payload with total mass of $25000 \mathrm{~kg}$. This scenario is shown in Figure 5. The initial velocity error is higher and takes a longer time for the controller to regulate the velocity. This can be more easily shown by comparing the closed-loop poles of this system to one in which mass is known. Figure 6 shows that underestimating the mass results in slower poles for closed loop system. ${ }^{\dagger \dagger}$

We observed a more critical situation when the mass of the truck is overestimated. In this scenario, as the discrepancy between the actual mass and estimated mass widens, the controller performance degrades rapidly and eventually can even result in an unstable closed-loop system. Figure 7 shows a scenario in which the controller is tuned for a fully loaded truck and is then used to control the truck without payload. Assuming large vehicle mass, the controller sends

\footnotetext{
$\overline{\dagger^{\dagger}}$ The poles are calculated for the linear system without constraints, but should give an idea of behaviour of the constrained system as well.
} 

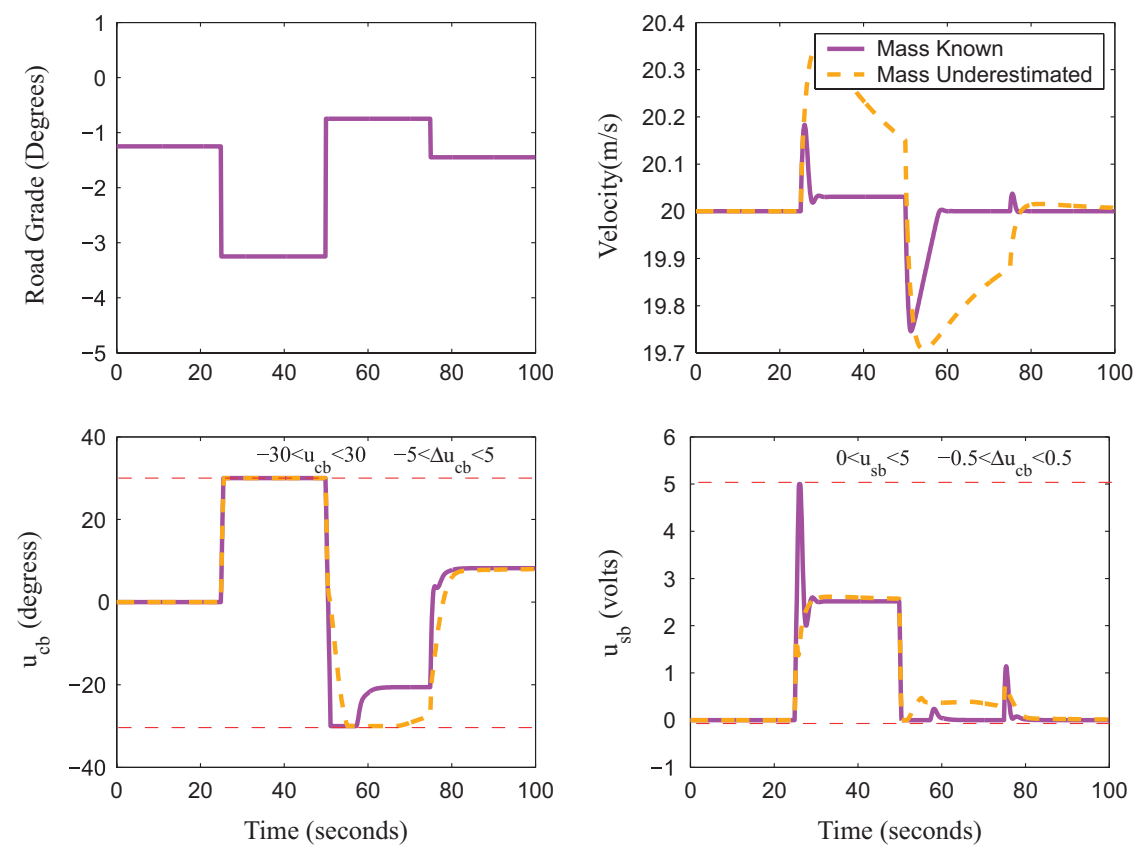

Figure 5. Velocity regulation when vehicle mass is underestimated. The actual and estimated mass are $25000 \mathrm{~kg}$ and $9000 \mathrm{~kg}$, respectively. The performance index weights are $Q=\operatorname{diag}(1,0.00002), S=\operatorname{diag}(0.01,0.1)$.

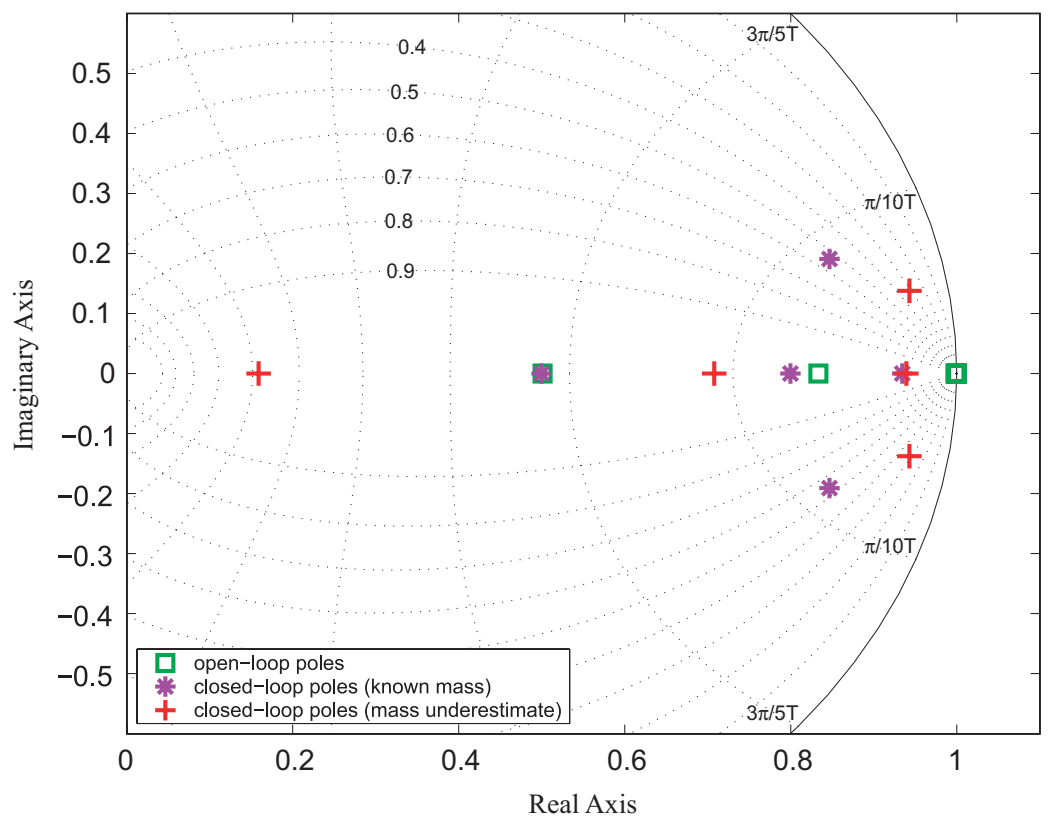

Figure 6. Pole locations for the open- and closed loop system, when mass is underestimated. 

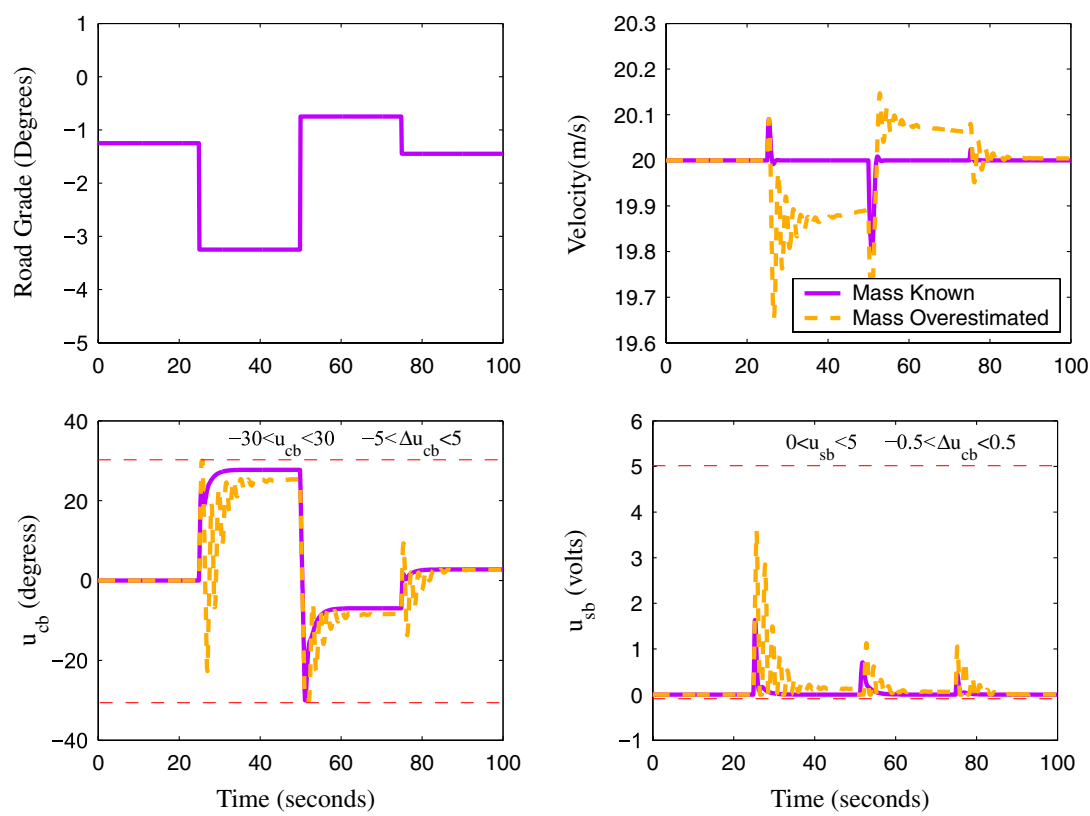

Figure 7. Velocity regulation when vehicle mass is overestimated. The actual and estimated mass are $9000 \mathrm{~kg}$ and $25000 \mathrm{~kg}$, respectively. The performance index weights are $Q=\operatorname{diag}(1,0.00002), S=\operatorname{diag}(0.01,0.1)$.

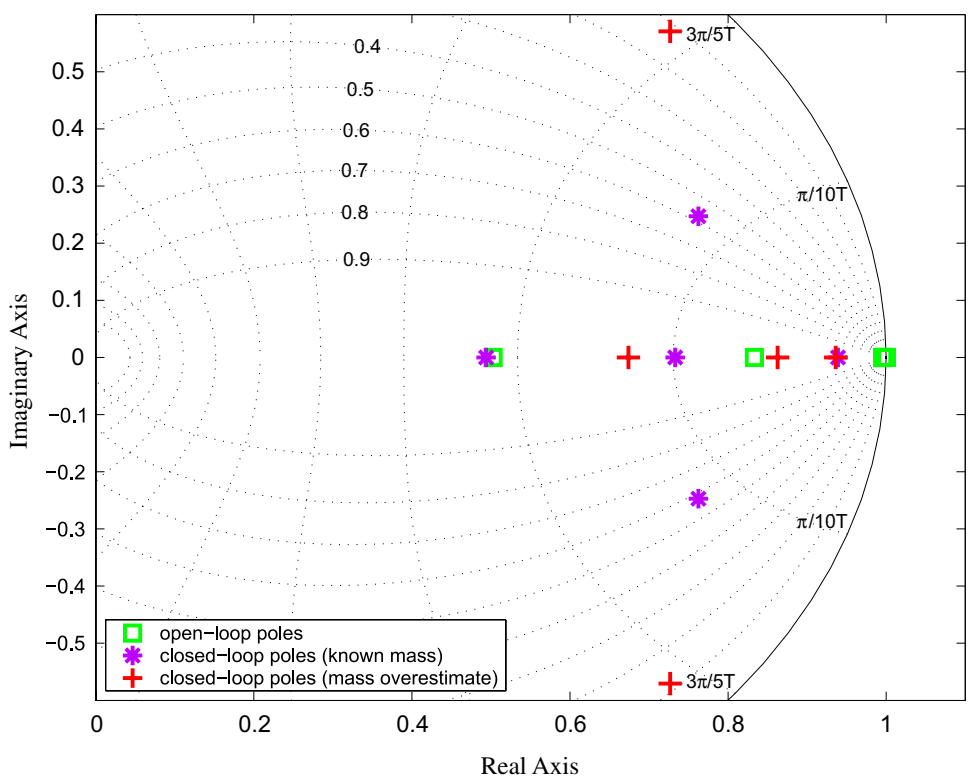

Figure 8. Pole locations for the open- and closed loop system, when mass is overestimated. 
large control commands which result in oscillatory closed-loop response. Figure 8 compares the pole locations of the closed-loop system with the case when mass is known. It can be seen that stability margin is small when mass is overestimated. When both grade and mass are unknown, the performance will degrade further.

Special care is necessary in the design of speed controller to prevent such scenarios which limit the use of longitudinal controls. In the next section we formulate an indirect adaptive controller which addresses the limitations of the fixed controller. The adaptation is based on an estimation scheme which estimates the vehicle mass and road grade simultaneously.

\section{THE ESTIMATION SCHEME}

A recursive least square algorithm with forgetting is used for estimation of vehicle mass and road grade disturbance. The estimation algorithm relies on the model of vehicle longitudinal dynamics and signals obtained from the vehicle CAN. Adding the nominal points, the vehicle dynamics equation (3) can be rewritten in the following form:

$$
\begin{aligned}
v(k+1)-v(k)= & \frac{T_{\mathrm{s}}}{M_{\mathrm{eff}}}\left(-2 k_{\mathrm{a}} v^{o} v(k)-T_{\mathrm{cb}} / r_{g}-T_{\mathrm{sb}} / r_{\mathrm{w}}+k_{\mathrm{a}}\left(v^{o}\right)^{2}\right) \\
& -T_{\mathrm{s}} g \frac{M}{M_{\mathrm{eff}}}\left(c_{\mathrm{rr}} \cos \beta+\sin \beta\right)
\end{aligned}
$$

Equation (20) can be written in the following parametric form:

$$
y=\phi^{\mathrm{T}} \theta, \quad \phi=\left[\begin{array}{ll}
\phi_{1} & \phi_{2}
\end{array}\right]^{\mathrm{T}}, \quad \theta=\left[\begin{array}{ll}
\theta_{1} & \theta_{2}
\end{array}\right]^{\mathrm{T}}
$$

where

$$
\theta=\left[\theta_{1}, \theta_{2}\right]^{\mathrm{T}}=\left[\frac{1}{M_{\mathrm{eff}}}, \frac{M}{M_{\mathrm{eff}}}\left(c_{\mathrm{rr}} \cos \beta+\sin \beta\right)\right]^{\mathrm{T}}
$$

is the parameter of the model to be determined and variables

$$
\begin{aligned}
y & =v(k+1)-v(k) \\
\phi_{1} & =T_{\mathrm{s}}\left(-2 k_{\mathrm{a}} v^{o} v(k)-T_{\mathrm{cb}} / r_{g}-T_{\mathrm{sb}} / r_{\mathrm{w}}+k_{\mathrm{a}}\left(v^{o}\right)^{2}\right) \\
\phi_{2} & =-T_{\mathrm{s}} g
\end{aligned}
$$

can be calculated based on measured signals from vehicle CAN and known vehicle parameters. The unknown parameter, $\theta_{1}$, depends on mass only and is constant and $\theta_{2}$ depends on road grade and is therefore time-varying.

In Reference [14], we employ a recursive least square scheme with vector-type forgetting, for estimation of vehicle mass and time-varying road grade. The recursive solution is:

$$
\hat{\theta}(k)=\hat{\theta}(k-1)+L(k)\left(y(k)-\phi^{\mathrm{T}}(k) \hat{\theta}(k-1)\right)
$$

where

$$
L(k)=P(k) \phi(k)=P(k-1) \phi(k)\left(\lambda I+\phi^{\mathrm{T}}(k) P(k-1) \phi(k)\right)^{-1}
$$


The covariance matrix $P$ is updated in the following way:

$$
P(k)=\Lambda^{-1}\left(I-L(k) \phi^{\mathrm{T}}(k)\right) P(k-1) \Lambda^{-1}
$$

where $\Lambda=\operatorname{diag}\left(\sqrt{\lambda_{1}}, \sqrt{\lambda_{2}}\right)$ and $0<\lambda_{1} \leqslant 1$ and $0<\lambda_{2} \leqslant 1$ are the forgetting factors for the first and second parameters, respectively. They are introduced to discard older information in favour of new information, so the algorithm can keep track of parameter variations. Smaller forgetting factors result in higher sensitivity to parameter variation. Choosing two values for $\lambda_{1}$ and $\lambda_{2}$ allows more degrees of freedom in the update of the two entries of $L(k)=\left[L_{1}(k), L_{2}(k)\right]$. In Reference [15], this algorithm is tested successfully with experimental data obtained from a heavy duty vehicle. It is shown that with persistent excitations, the estimator performs well in presence of realistic signal noise.

\section{SIMULATION RESULTS: WITH ADAPTATION}

The estimator is implemented in parallel with the predictive control module and updates the model at each sampling time based on the latest estimate of mass. Also the grade estimate is used for feedforward control. Our assumption is that no prior knowledge of mass or grade is available. Before initializing the recursive estimator, a batch least square estimation is performed, using the initial measurements. To ensure that level of excitations is sufficient for batch estimation, the following persistent excitation condition [16] is checked, till it is fulfilled:

$$
\left.\sum_{i=1}^{k}\left(\phi(i) \phi(i)^{\mathrm{T}}\right)\right)>\rho I_{2 \times 2}
$$

with $\rho=0.01$. After obtaining the initial batch estimate, estimation is continued recursively using the recursive least square with vector-type forgetting. The forgetting factor for grade was chosen at 0.5 to keep the estimator sensitive to variations in grade. The mass is constant but we chose the forgetting factor slightly smaller than 1 at 0.95 . This choice expedites convergence of mass to its actual value in some scenarios. This adaptive controller tested successfully in simulations of different scenarios. Figure 9 shows one of the critical cases, in which overestimation of mass and selection of aggressive penalty weights result in rapid oscillations in closed-loop response, in absence of adaptation. When the adaptive scheme is used, the performance improves noticeably, and the oscillatory behaviour is reduced. Figure 10 shows the estimation results. Mass and grade are both estimated well. In fact the parameter estimation improved as more excitation was provided to the system, through the initial oscillatory behaviour following the step change in grade and saturation of both retarders. The initial velocity was reduced dramatically after 50 sampling instants $(5 \mathrm{~s})$. Although the results are very promising from stability perspective, implementation on a truck will allow us to assess drivability or effects of driveline dynamics. Figures 11 and 12 show the same when the mass was underestimated. The adaptation now improves the speed of velocity tracking. Note that the mass estimate does not asymptotically converge to its actual value in this case, this may be due to lower oscillation levels in response when mass is underestimated, which suggests that persistent excitation conditions may be only poorly met in some periods of the simulation. 

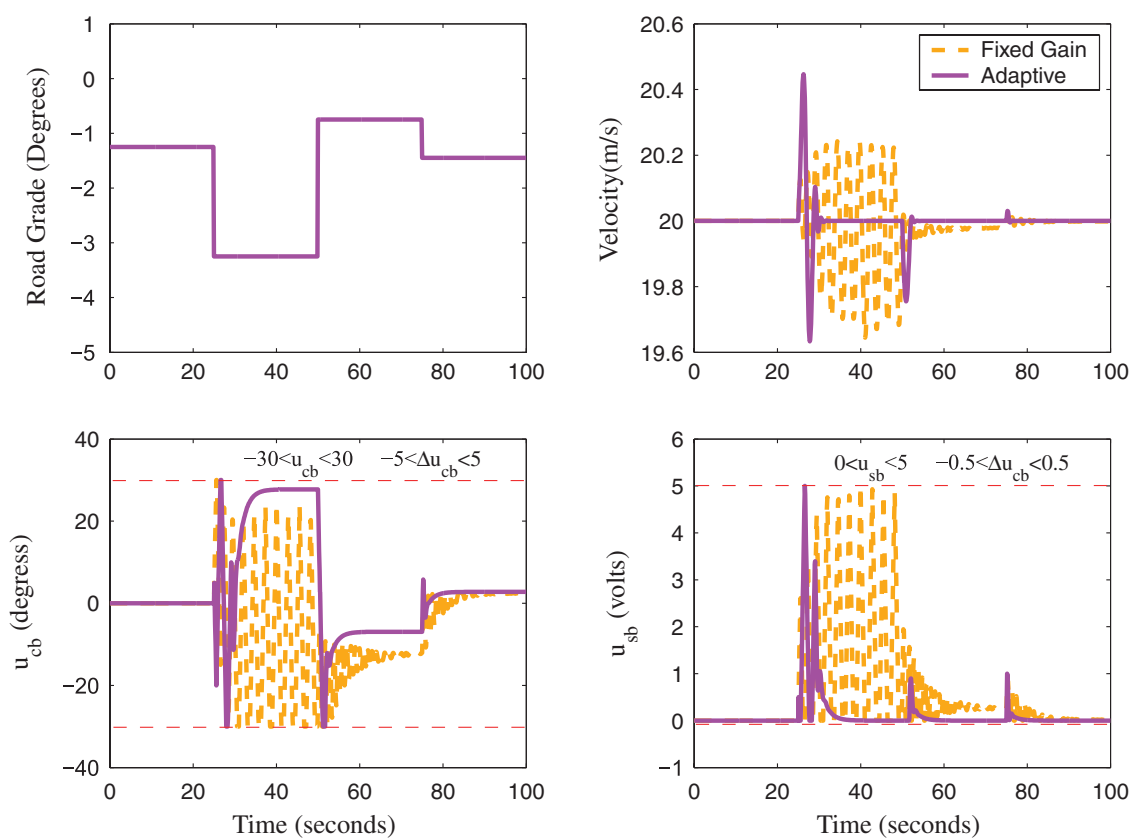

Figure 9. Comparison of the adaptive and fixed gain schemes when mass is overestimated. The actual and initial estimate of mass are $9000 \mathrm{~kg}$ and $25000 \mathrm{~kg}$, respectively. The performance index weights are $Q=\operatorname{diag}(5,0.00002), S=\operatorname{diag}(0.01,0.1)$.
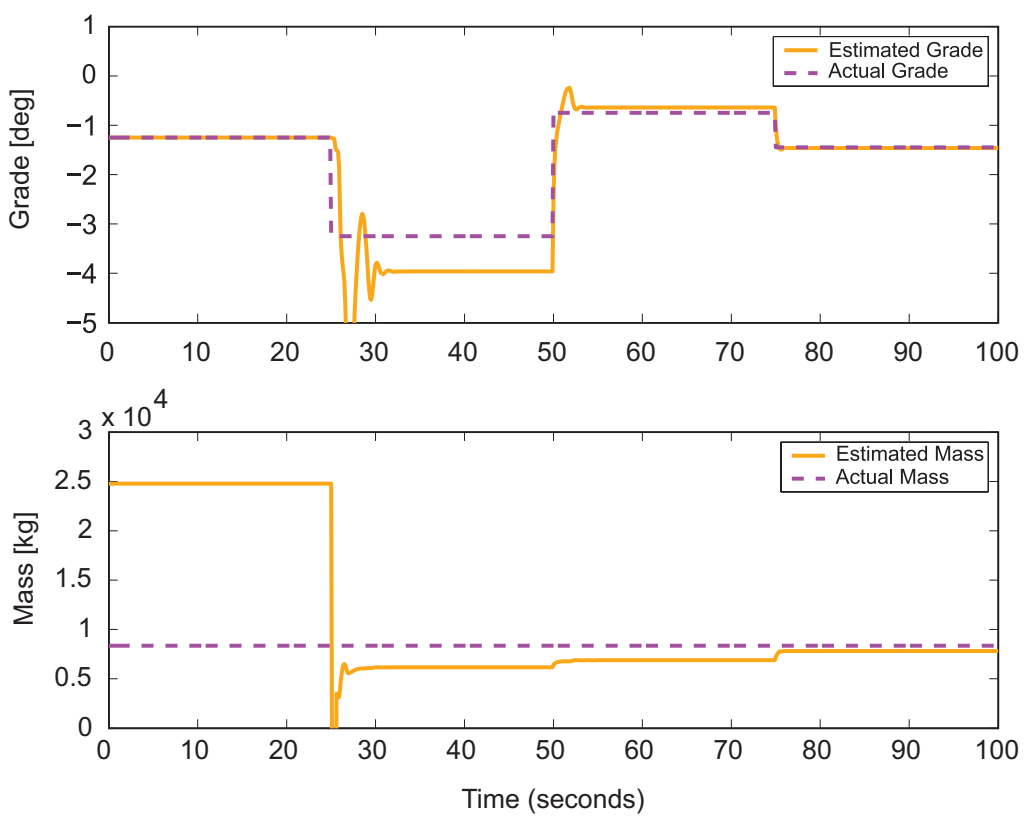

Figure 10. Simultaneous estimation of mass and grade when mass was overestimated. Forgetting factors for mass and grade are 0.95 and 0.5 , respectively. 

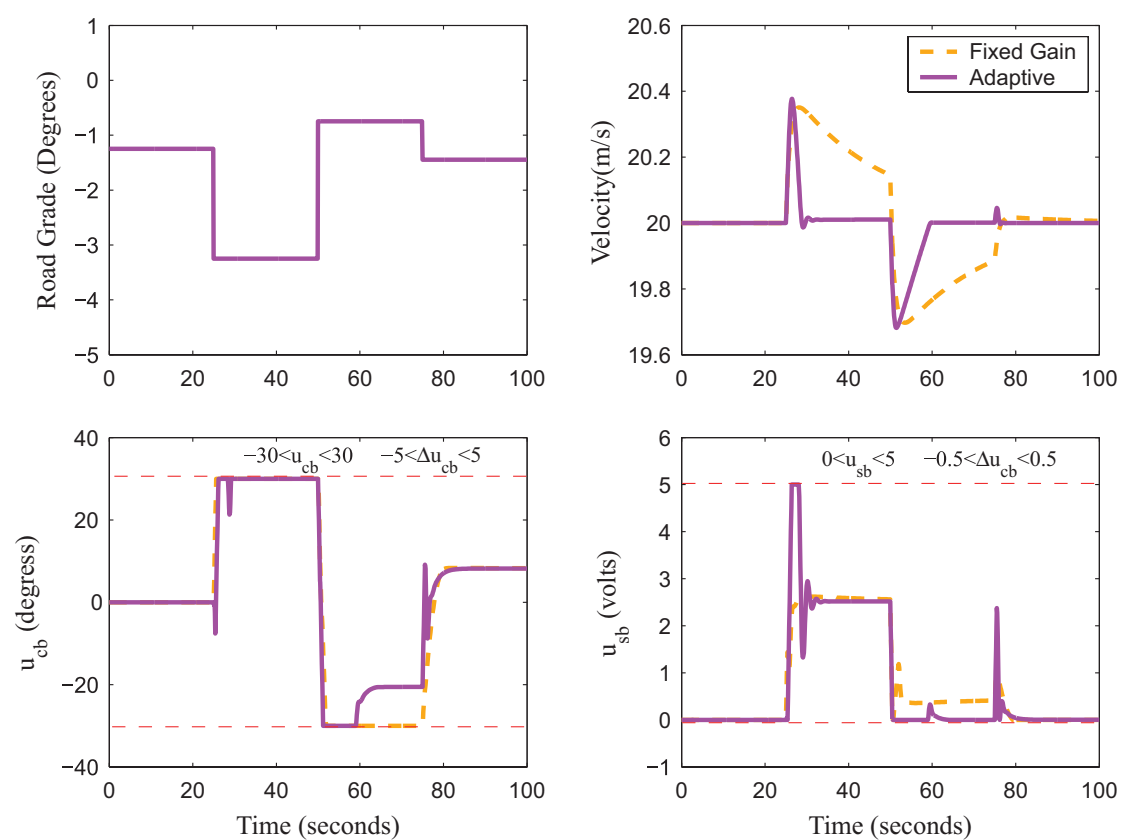

Figure 11. Comparison of the adaptive and fixed gain schemes when mass is underestimated. The actual and initial estimate of mass are $25000 \mathrm{~kg}$ and $9000 \mathrm{~kg}$, respectively. The performance index weights are $Q=\operatorname{diag}(5,0.00002), S=\operatorname{diag}(0.01,0.1)$.
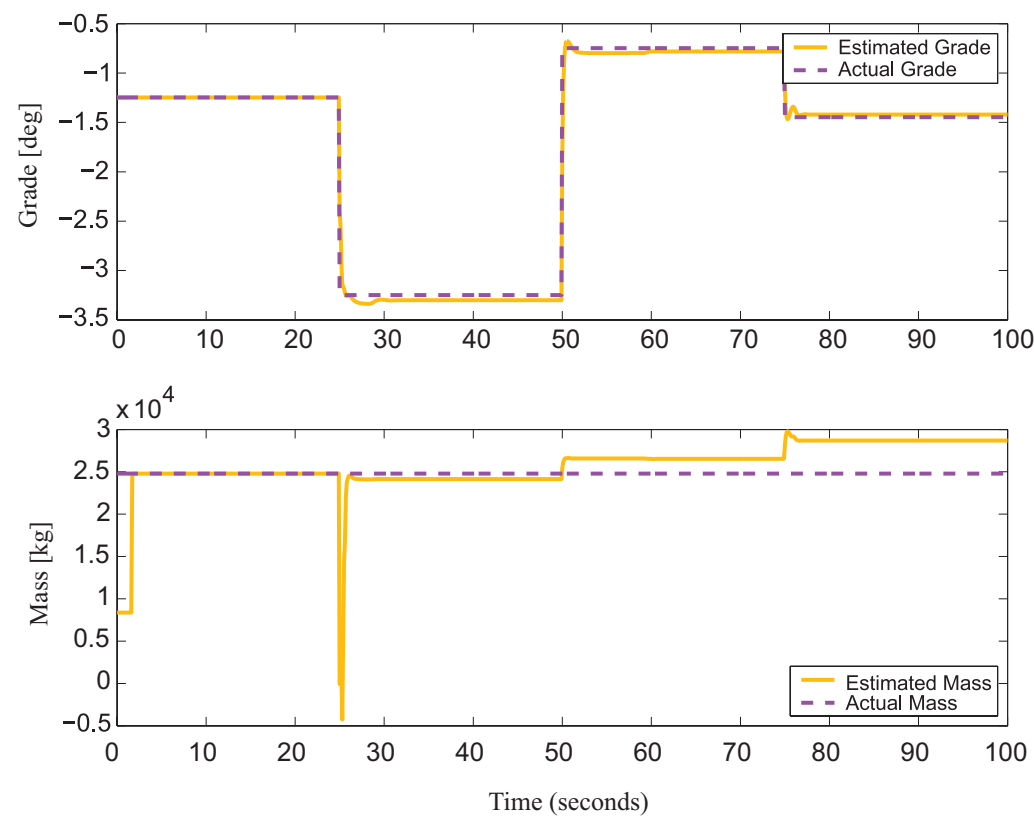

Figure 12. Simultaneous estimation of mass and grade when mass was underestimated. Forgetting factors for mass and grade are 0.95 and 0.5 , respectively. 


\section{CONCLUSIONS}

In this paper we formulated the velocity tracking problem of heavy duty truck in a model predictive control framework, which enable us to explicitly account for actuator saturation limits in the control design. The controller co-ordinates use of compression brakes and friction brakes on downhill slopes. The use of friction brake was minimized by penalizing its steady-state use. With knowledge of the plant parameters and dynamics, good performance was obtained in closed loop. We showed with simulation analysis that uncertainty in vehicle mass degrades the closed-loop response. Unknown road disturbance (grade) was another limiting factor to the performance of the controller.

To rectify such problems, a recursive least square scheme with forgetting factor was used in an indirect adaptive scheme. The estimation scheme runs in parallel with the controller and updates the model at each step based on the new estimate of mass. Grade estimates are also used in feedforward control. The estimator converged quickly and improved the closed-loop performance. Addition of grade estimates in feedforward control contributed to rapid regulation of velocity. The robustness of the algorithm under higher order driveline and tire dynamics will be investigated as future work. Ultimately the algorithm presented needs to be integrated seamlessly with anti-lock braking subsystems.

The MPC design has a few advantages over other possible control methods. In particular MPC can explicitly handle input constraints, something that is not directly addressed in a method like PID control and extra measures such as anti-windup are needed. A Lyapunovbased direct adaptive scheme has been used in the past for a similar problem with unknown mass and grade; however MPC has the advantage that it 'optimally' splits the torque between two braking mechanism, which is not straightforward in the Lyapunov-based scheme.

\section{ACKNOWLEDGEMENTS}

This work was supported in part by the California Department of Transportation through the California PATH Program under TO 4234. The authors wish to thank Professor Jing Sun of University of Michigan for her feedback on the adaptive control section.

\section{REFERENCES}

1. Shladover S. Review of the state of development of advanced vehicle control systems (avcs). Vehicle System Dynamics 1995; 24:551-595.

2. Vahidi A, Eskandarian A. Research advances in intelligent collision avoidance and adaptive cruise control. IEEE Transactions on Intelligent Transportation Systems 2003; 4(3):143-153.

3. Yanakiev D, Kanellakopoulos I. Longitudinal control of automated CHV's with significant actuator delays. Proceedings of 36th IEEE Conference on Decision and Control, 1997.

4. Druzhinina M, Stefanopoulou A. Speed control experiments for commercial heavy vehicles with coordinated friction and engine compression brakes. Proceedings of the American Control Conference, Anchorage, AK, U.S.A., 2002.

5. Moklegaard L, Stefanopoulou AG, Schmidt J. Transition from combustion to variable compression braking. $S A E$ Paper No. 2000-01-1228, 2000.

6. Druzhinina M, Moklegaard L, Stefanopoulou A. Speed gradient approach to longitudinal control of heavy-duty vehicles equipped with variable compression brake. IEEE Transactions on Control Systems Technology 2001; 10: $209-220$. 
7. Druzhinina M, Stefanopoulou A, Moklegaard L. Adaptive continuously variable compression braking control for heavy-duty vehicles. ASME Dynamic Systems, Measurement and Control 2002; 124:406-414.

8. Moklegaard L, Druzhinina M, Stefanopoulou A. Brake valve timing and fuel injection: a unified engine torque actuator for heavy-duty vehicles. Vehicle System Dynamics 2001; 36(2-3):179-201.

9. Lu X-Y, Hedrick K. Longitudinal control design and experiment for heavy-duty trucks. Proceedings of the American Control Conference, Denver, CO, U.S.A., 2003; 36-41.

10. Lu X-Y. Email communication with Xiao-Yun Lu from PATH. July 2003.

11. Maciejowski JM. Predictive Control with Constraints. Prentice-Hall: Essex, England, 2002.

12. Vahidi A, Stefanopoulou A, Peng H. Recursive least squares with forgetting for online estimation of vehicle mass and road grade: theory and experiments. Journal of Vehicle System Dynamics 2005; 43:31-57.

13. Joo S, Lu XY, Hedrick JK. Longitudinal maneuver design in coordination layer for automated highway system. Proceedings of the American Control Conference, Denver, CO, U.S.A., 2003; 42-47.

14. Vahidi A, Druzhinina M, Stefanopoulou A, Peng H. Simultaneous mass and time-varying grade estimation for heavy-duty vehicles. Proceedings of the American Control Conference, Denver, CO, U.S.A., 2003.

15. Vahidi A, Stefanopoulou A, Peng H. Experiments for online estimation of heavy vehicle's mass and time-varying road grade. Proceedings of IMECE, Washington, DC, U.S.A., 2003.

16. Astrom A, Wittenmark B. Adaptive Control (2nd edn). Addison-Wesley: Reading, MA, 1994. 